\title{
Myositis ossificans degenerating into extra-skeletal osteosarcoma: a rare disorder, which degenerates more rarely: case report
}

\begin{abstract}
Myositis ossificans is a benign lesion characterized by focal heterotopic soft tissue ossification and occurring in young people generally after localized trauma. It can mimic osteosarcoma but it rarely degenerates. We report a case of a patient with myositis ossificans which degenerates into extra-skeletal osteosarcoma.
\end{abstract}

Keywords: ossificans myositis, extra skeletal osteosarcoma
Volume 4 Issue 2 - 2020

\author{
Sara Dahhouki,' Khadija Issoual,' Zakia \\ Douhi,' Hanane Baybay,' Salim Gallouj,' \\ Fatima Zahra Mernissi, ${ }^{\prime}$ Tarik Youssoufi, ${ }^{2}$ \\ Taoufik Harzy, ${ }^{2}$ Chaymae Hajjar, ${ }^{3}$ Mustapha \\ Maaroufi, ${ }^{3}$ Mouna Rimani ${ }^{4}$ \\ 'Dermatology department, CHU Hassan II, Fez, Morocco \\ ${ }^{2}$ Rheumatology department, CHU Hassan II, Fez, Morocco \\ ${ }^{3}$ Radiology department, CHU Hassan II, Fez, Morocco \\ ${ }^{4}$ Laboratory of pathology Hassan Rabat, Morocco
}

Correspondence: Sara Dahhouki, Dermatology department, CHU Hassan II, Fez, Morocco, Email dahhoukisara@gmail.com

Received: March 04, 2020 | Published: May 25, 2020

\section{Introduction}

Myositis ossificans, defined by heterotopic ossification of striated muscles; it is a rare and benign disorder which degenerates less than $1 \% .{ }^{1,2}$ We report a case of extra-skeletal osteosarcoma that developed from myositis ossicans.

\section{Case report}

A 40-year old woman presented, 5 years before her consultation, following a neglected trauma, a swelling of the right thigh gradually increasing in volume, non-painful, without alteration of the general state. Magnetic resonance imaging, 5 years ago, showed a significant increase in thigh muscles. The signal was hyper intense on $\mathrm{T}_{2}$, with calcifications and cystic areas. Histology exhibited large ossification foci, made up of osteoid trabeculae with striated muscles largely ossified and ballooned muscle fibers that are penetrated by an inflammatory infiltrate. Immuno histochemistry had confirmed the diagnosis: anti-Smooth Muscle Actin and anti-vimentine antibodies were positive, and the index of Ki67 proliferative activity was positive in $15 \%$. After 4 years, evolution was marked by a very rapid increase of volume of right thigh, with necrotizing ulcerations and altered general condition evident just in one month. Clinical examination revealed a mass, covering the entire right thigh, hard, fixed and painful. The surface was covered by multiple hard, fixed sub cutaneous nodules with multiple ulcerations with a necrotic centre. Histology showed a tumour proliferation made of spindle-shaped cells arranged in bundles of anarchic direction, with anisokaryosis and high mitotic index. Immuno-histochemistry objectified a positive anti-Vimentin antibody. CT scan showed a large, heterogeneous and calcified tissular mass invading muscle, fat and subcutaneous tissue. The extension workup showed secondary lung localization. Biology revealed inflammatory anemia as well as a hyponatremia and hypocalcemia that were resistant to treatment. The patient died after 1 months of progression.

\section{Discussion}

Myositis ossificans is a benign disorder characterized by abnormal heterotopic bone formation that is essentially metaplasia of the intramuscular connective tissue resulting in extra osseous bone formation. ${ }^{1}$ Thigh involvement is the most common, ${ }^{2}$ like our patient. Histologically, Myositis ossificans evolves in three phases: an active mesenchymal proliferation of fibroblasts, followed by an appearance of heterotopic osteoblasts and the creation of a fibrous capsule, which constitutes a "Zone Phenomenon", with three distinct zones: central, consisting of mesenchymal tissue and sometimes fibroblast mitosis, hemorrhage and necrosis, intermediate with osteoblasts and islets of immature bone and an outer zone, with trabeculae of mature bone and centrifugal maturation. ${ }^{3}$ The literature contains several case reports of instances in which myositis ossificans has mimicked malignant neoplasm clinically and even histologically. Diagnoses such as osteogenic sarcoma, Ewing's sarcoma, neuroblastoma, Wilms' tumor, lymphoma, myosarcoma, and fibrosarcoma have been mistakenly proffered after microscopic examination of apparently relatively undifferentiated foci of myositis ossificans, ${ }^{4}$ but malignant transformation of myositis ossificans is extremely rare. ${ }^{5}$ Its incidence is difficult to specify because of its rarity and the difficulty to distinguish circumscribed ossifying myositis, secondarily degenerated, from an initially misdiagnosed sarcoma. ${ }^{4-7}$ The prognosis is unfortunate ${ }^{6}$ as such as the case of our patient (Figures 1-4). 


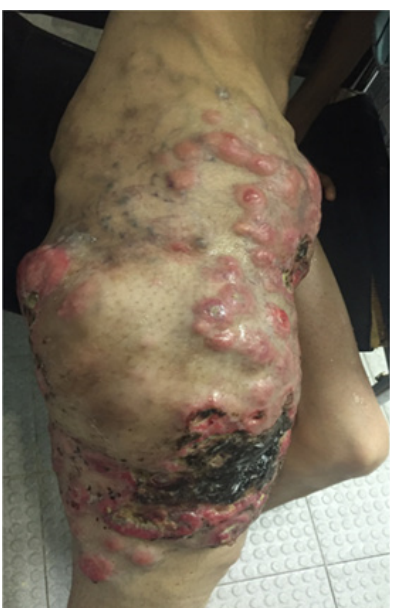

Figure I Extra-skeletal osteosarcoma of the thigh muscles.

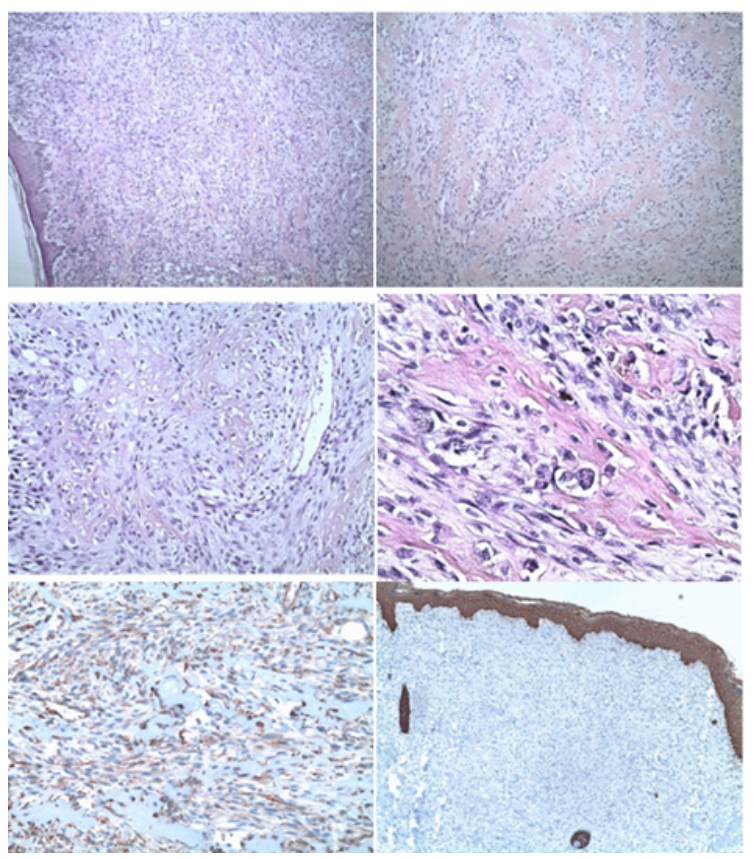

Figure 2 Tumour proliferation sarcomatoid of the dermis and hypodermis with spindle cell, globoid and osteoids, $(A, B)$ with cellular atypia consisting of anisokaryosis and mitosis. (C,D) These cells express diffusely vimentin (E,F), and do not express cytokeratin.
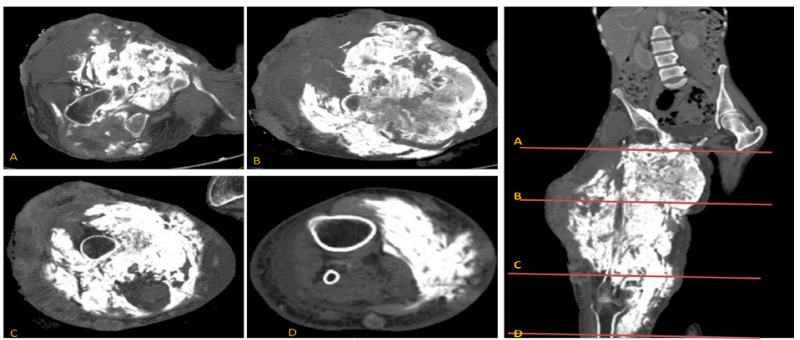

Figure 3 MRI: large, heterogeneously calcified predominantly hypo dense tissue mass heterogeneously enhanced after contrast containing areas of liquefaction measuring $28 \times 20 \times 50 \mathrm{~mm}$. This mass invades muscle, fat and skin tissue with bone lysis of the femoral bone in localized areas. Above it reaches the iliac psoas muscle and the right muscle of the right abdomen, sheathing the common homolateral femoral pedicle, which remains permeable. Below it reaches the anteromedial compartment of the right leg.
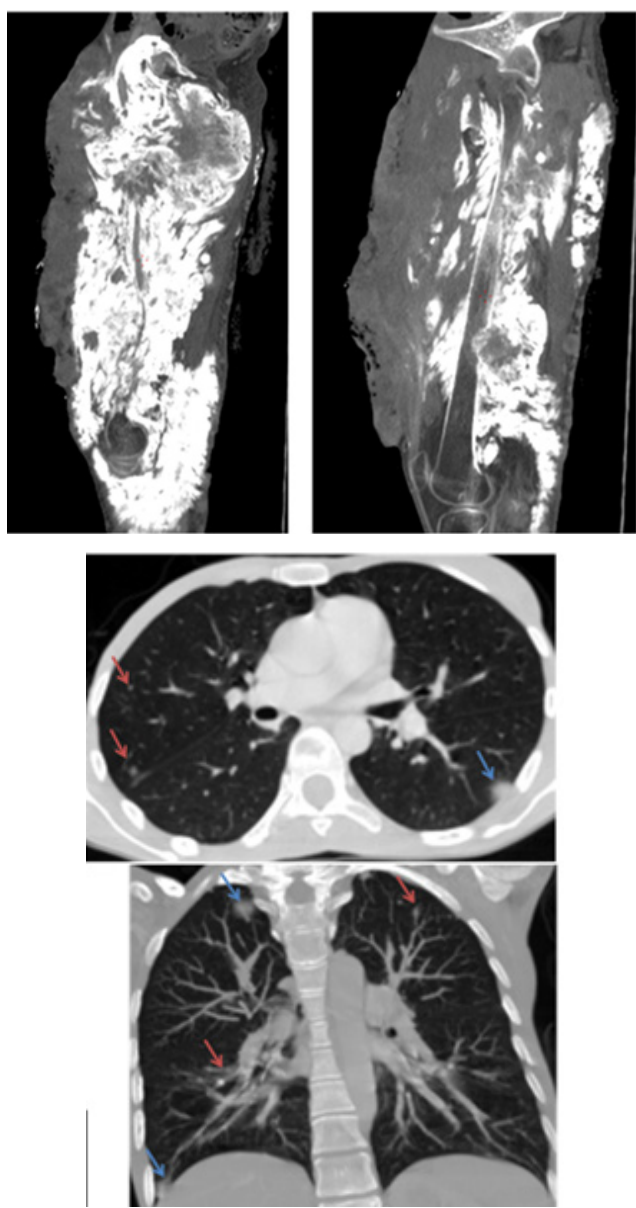

Figure 4 CT scan:(A-B) heterogeneous hyper dense mass in the thigh muscles. (C-D) Masses (blue arrow) intra parenchymal and sub pleural scattered in the 02 pulmonary fields with speculate contours-secondary locations.

\section{Conclusion}

Although ossificans myositis remains a diagnosis of elimination, once it has been diagnosed, clinical and radiological monitoring is necessary, in order to avoid the risk, even minimal, of sarcomatous degeneration.

\section{Conflicts of interest}

The author declares that there is no conflicts of interests.

\section{Acknowledgments}

None.

\section{Funding}

None.

\section{References}

1. Pohlodek K, janík M, Mečiarová I, et al. Pseudomalignant myositis ossificans in the breast: A case report. Mol Clin Oncol. 2018;8(6):749752.

2. Fnini S, Arssi M, Hassoun J, et al. Largab Myosite ossifiante circonscrite de l'avant-bras. Chirurgie de la main. 2009;28(6):370-373.

3. Bernard M, Coumbaras M, Zeitoun F, et al. Le Hir P. Spectre radiologique évolutif de la myosite ossifiante circonscrite. J Radiol. 2003;84(1):54-56. 
4. Shanoff L, Spira M, Hardy SB. Myositis Ossificans: Evolution to Osteogenic Sarcoma: Report of a Histologically Verified Case. American Journal of Surgery. 1967;113(4):537-541.

5. Konishi Ei, Kusuzaki K, Murata H, et al. Extraskeletal osteosarcoma arising in myositis ossificans. Skeletal Radiol. 2001;30(1):39-43.
6. Alouini-Mekki R, El Mhabrech H, Hasni I, et al. La myosite ossifiante circonscrite : apport de l'imagerie. J Radiol. 2007;88(5):663-668.

7. Bronfen $\mathrm{C}$, Touzet $\mathrm{Ph}$, Peuchmaur $\mathrm{M}$, et al. Myosite ossifiante non traumatique chez l'enfant. Revue de la littérature : à propos d'un cas simulant une tumeur maligne. Revue de chirurgie orthopédique et réparatrice de l'appareil moteur. 1993;79(3):229-234. 